\title{
Structure of the Silica/Divalent Electrolyte Interface: Molecular Insight into Charge Inversion with Increasing $\mathrm{pH} \dagger$
}

Mokhtar Rashwan, ${ }^{1 \ddagger}$ Benjamin Rehl, ${ }^{1 \ddagger}$ Adrien Sthoer, ${ }^{2}$ Akemi M. Darlington, ${ }^{1}$ Md. Shafiul Azam, ${ }^{3}$ Hongbo Zeng, ${ }^{3}$ Qingxia Liu, ${ }^{3}$ Eric Tyrode, ${ }^{2 *}$ Julianne M. Gibbs ${ }^{1 *}$

${ }^{1}$ Department of Chemistry, University of Alberta, Edmonton, Alberta T6G 2G2, Canada. julianne.gibbs@ualberta.ca

${ }^{2}$ Department of Chemistry, KTH Royal Institute of Technology, SE-10044 Stockholm, Sweden. tyrode@kth.se

${ }^{3}$ Department of Chemical and Materials Engineering, University of Alberta, Edmonton, Alberta T6G 2G2, Canada

$\uparrow$ Electronic supplementary information (ESI) available. ppp-VSF spectra collected at an extended spectral range, fitting procedure, summary of fitted values, zeta potential replicates.

† MR and BR contributed equally to this work. 


\section{TOC GRAPHIC}

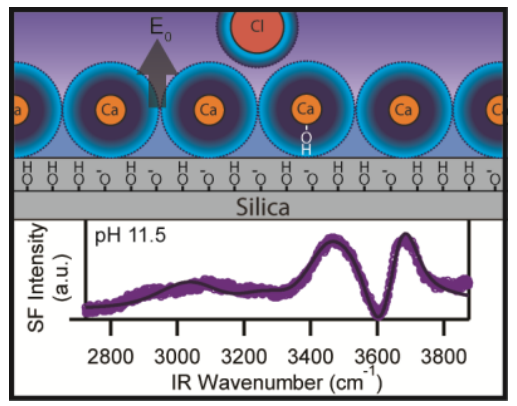

\section{ABSTRACT}

The molecular origin of overcharging at mineral oxide surfaces remains a cause of contention within the geochemistry, physics, and colloidal chemistry communities owing to competing "chemical" vs "physical" interpretations. Here, we combine vibrational sum frequency spectroscopy and streaming potential measurements to obtain molecular and macroscopic insights into the $\mathrm{pH}$-dependent interactions of calcium ions with a fused silica surface. In $100 \mathrm{mM} \mathrm{CaCl}_{2}$ electrolyte, we observe evidence of charge neutralization at $\mathrm{pH} \sim 10.5$, as deducted from a minimum in the interfacial water signal. Concurrently, adsorption of calcium hydroxide cations is inferred from the appearance of a spectral feature at $\sim 3610 \mathrm{~cm}^{-1}$. However, the interfacial water signal increases at higher $\mathrm{pH}$, while adsorbed calcium hydroxide appears to remain constant, indicating that overcharging results from hydrated $\mathrm{Ca}^{2+}$ ions present within the Stern layer. These findings suggest that both specific adsorption of hydrolyzed ions and ion-ion correlations of hydrated ions govern silica overcharging with increasing $\mathrm{pH}$. 


\section{INTRODUCTION}

One question that has long puzzled surface scientists is why mineral oxides at $\mathrm{pH}$ above their point-of-zero charge can exhibit a reversal in the effective charge of their corresponding electric double layer from negative to positive in the presence of certain ions. ${ }^{1-12}$ Electrostatic models based on mean field approximations of ion-surface interactions cannot account for such overcharging as the driving force for concentrating ions at an interface is zero once the system has been neutralized. ${ }^{8,12}$ However, since the 1970 s, experimental evidence of overcharging has been reported. ${ }^{9-10}$ For example, surface adhesion experiments have shown that divalent ions can lead to attractive interactions between mineral surfaces at $\mathrm{pH}$ above their respective points-of-zero charge. ${ }^{13-14}$ Similarly, electrokinetic measurements on both mineral colloids and surfaces in the presence of divalent or trivalent ions have yielded positive zeta potentials at a $\mathrm{pH}$ where the surfaces should be negative. ${ }^{2,4,11,15-16}$ The driving force for overcharging has been attributed to either "physical" interactions like ion-ion/ion-site correlations and dispersion forces (favoured by physicists) or "chemical" interactions that involve the formation of specific surface complexes (favoured by chemists). ${ }^{8,12,17-18}$ However, separating the effects of such physical or chemical interactions has proven particularly difficult for mineral oxides and multivalent ions based on the tendency of the latter to form different hydrolyzed species at higher $\mathrm{pH}^{8}{ }^{8}$ Indeed, the quest to determine whether it is hydrated divalent ions that lead to overcharging through dispersion forces (an outer sphere interaction) or it is the hydrolyzed ionic species adsorbing specifically (an inner sphere or ion-pair interaction) drove much effort in surface science, and in particular that of the late Johannes Lyklema. ${ }^{11-12}$

This phenomenon of overcharging reveals that the properties of charged mineral oxides can be drastically altered when aqueous multivalent ions are present by effectively converting a 
negatively charged surface into a positive one, even at high $\mathrm{pH} \cdot{ }^{1-2,4,7}$ Given that interactions of ions with mineral oxides have significant real-world consequences, they have also been explored in the context of soil remediation, ${ }^{19}$ mineral beneficiation and agglomeration, ${ }^{20}$ water treatment, ${ }^{21}$ and oil sand tailings treatment. ${ }^{13,22-24}$ Yet, while numerous studies have examined the effect of divalent ions on the properties of silica as well as other mineral oxides, the molecular interfacial structure under conditions of overcharging remains largely ${ }^{7}$ a mystery. Very recently, Legg and co-workers observed charge reversal of a mica surface with $\mathrm{Al}^{3+}$ ions using $\mathrm{AFM}$ and streaming potential together with AIMD simulations. They found that the surface potential of mica reverses from negative to positive with increasing $\mathrm{pH}$ due to specific adsorption of hydrolyzed species $\left(\mathrm{Al}(\mathrm{OH})^{2+}\right.$ and $\left.\mathrm{Al}(\mathrm{OH})_{2}{ }^{+}\right) \cdot{ }^{16}$ Another method that is intrinsically surface selective and sensitive to molecular speciation is the second-order non-linear optical technique vibrational sum frequency spectroscopy (VSFS). VSFS has been extensively used to investigate the amount of ordered water at the silica/water interface, ${ }^{25-31}$ which can be used to report on the interfacial potential within the electric double layer. ${ }^{32-34}$ Moreover, VSFS should be able to disentangle the presence of hydrated ions in the electrical double layer from hydrolyzed ions owing to the appearance of new $\mathrm{OH}$ oscillators in the spectra. Nevertheless, such studies have not been forthcoming until now.

Previous studies focusing on divalent ion interactions show that the amount of ordered water at the silica/water interface is attenuated compared to monovalent ions. However, these initial studies were limited to low salt concentrations, resulting in still significant amounts of ordered water at the silica mineral interface. ${ }^{35}$ More recently, the effect of molar concentrations of alkali and alkaline earth chlorides on the silica/aqueous interface at $\mathrm{pH} 6$ were studied by Chou, Bertram and co-workers. ${ }^{36}$ The authors found that the SF signal from interfacial water molecules dropped drastically upon the addition of $4 \mathrm{M}$ aqueous $\mathrm{CaCl}_{2}$ solution. They attributed the decrease 
in intensity to the formation of an ion pair between a deprotonated silanol and $\mathrm{Ca}^{2+}$ cation, with a water molecule acting as a bridge. ${ }^{36}$ However, the concentrations that led to the effect were very high, providing a barrier to industrial use. Additionally, the proposed mechanism of calcium interaction with silica did not address the role of other influential interfacial properties like the surface potential, nor did it explain from a symmetry perspective why such a surface complex would result in less signal.

Here we combine VSFS with streaming potential measurements to determine the impact of $100 \mathrm{mM}$ calcium ions on the interfacial water structure and other hydroxide species present at the mineral oxide interface over a wide range of $\mathrm{pH}$. We observe that the SF signal intensity drops when increasing the $\mathrm{pH}$ from 6 to 10.5 and increases thereafter, in direct contrast to what has been observed for monovalent ions under similar conditions. ${ }^{25,29,31,37}$ Furthermore, at higher $\mathrm{pH}$, we see evidence of calcium hydroxide species, interacting with the negative silica surface, providing the first clear evidence of hydrolyzed ions by VSFS at this interface. The results are explained by charge neutralization and subsequent charge reversal from negative to positive with increasing $\mathrm{pH}$. The evolution of the water intensity with $\mathrm{pH}$ as well as the presence of the calcium hydroxide cation at the interface are discussed within the context of current theories of overcharging at mineral oxide interfaces. ${ }^{1-6,8-12}$

\section{RESULTS AND DISCUSSION}

In our experiments, the SF signal is generated by temporally and spatially overlapping visible and infrared pulsed laser at the silica/water interface in a non-collinear reflection geometry. Due to the selection rules of SFG within the electric dipole approximation, SF signal is only generated in media lacking inversion symmetry, a property inherent to interfaces. ${ }^{38}$ For VSFS, this signal is enhanced when the incident electric field in the IR range is on resonance with a vibrational 
mode of the media that is both Raman and IR active. Furthermore, at a charged interface, the

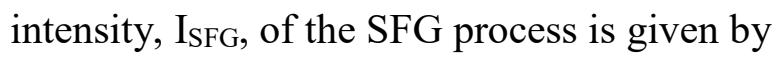

$I_{S F G} \propto\left|\chi^{(2)}+\chi^{(3)} \Phi_{0}\right|^{2} I_{V i S} I_{I R}$

eq. 1

Where $I_{\text {vis }}$ and $I_{I R}$ are the incident intensities of the visible and infrared light fields, respectively. The second order nonlinear susceptibility of the medium, $\chi^{(2)}$, describes ordered interfacial species immediately adjacent to the surface like water molecules that make up the Stern layer that hydrate the silica surface as well as specifically adsorbed ions. Surface silanols and other hydroxyl species on resonance in the measured IR frequency range also contribute to $\chi^{(2)}$. The second term containing $\chi^{(3)}$, the third-order susceptibility, stems from the net alignment of water in the diffuse layer that results from the charged surface and its corresponding interfacial potential $\Phi_{0}$. Here we neglect the z-dependence (surface normal) of $\chi^{(3)} \Phi_{0}$ due to the short Debye screening length resulting from the high salt concentrations used in this study. ${ }^{32-33,39-44}$

The starting point of our discussion is the VSF spectra measured with the ppp-polarization combination ( $p$ - $\mathrm{I}_{\mathrm{SFG}}, p-\mathrm{I}_{\mathrm{vis}}$ and $p$ - $\mathrm{I}_{\mathrm{IR}}$ ) at the silica/aqueous calcium chloride interface as a function of $\mathrm{pH}$ from neutral to basic, as shown in Fig. 1a. This polarization combination was selected as it is known to be most sensitive to the amount of ordered water along the z-direction (surface normal) which is the direction of the static surface field set up by the charged interface. ${ }^{7}$ The composition of the aqueous phase was $100 \mathrm{mM} \mathrm{CaCl}_{2}$ with $10 \mathrm{mM} \mathrm{NaCl}$ background electrolyte to account for changes in sodium concentration upon $\mathrm{pH}$ adjustment with $\mathrm{NaOH}_{(\mathrm{aq})}$. At the initial $\mathrm{pH}$ of $\sim 6$, two broad features around $3200 \mathrm{~cm}^{-1}$ and $3400 \mathrm{~cm}^{-1}$ were observed. These bands are attributed to water molecules with a preferred orientation at a hydrophilic interface, ${ }^{29,} 32-33,35,42,45-47$ as well as molecules in the diffuse layer aligned by the surface electric field. In stark contrast to previous 
studies in the presence of monovalent salts such as sodium chloride, ${ }^{25,29,31,37}$ the SF signal decreased as the $\mathrm{pH}$ was raised to 10.5 (Fig. 1a). Upon further increasing the $\mathrm{pH}$, the overall SF intensity started to increase, particularly at the higher frequencies. This unusual behaviour is highlighted in Fig. 1b, where the integrated ppp-SF intensity as a function of $\mathrm{pH}$ for the $100 \mathrm{mM}$ $\mathrm{CaCl}_{2}$ system is compared with our previous work using $100 \mathrm{mM}$ and $500 \mathrm{mM} \mathrm{NaCl}$ solutions. ${ }^{25}$, 37 For the monovalent salts, the increase in SF intensity with increasing $\mathrm{pH}$ was attributed to the larger absolute interfacial potentials resulting from the increased deprotonation of the silica substrate. ${ }^{45,48}$ However, as shown in Fig. $1 \mathrm{~b}$, for $100 \mathrm{mM} \mathrm{CaCl}_{2}$ at $\mathrm{pH} 10.5$ the integrated intensity decreased to just $18 \%$ of its value at $\mathrm{pH} 6$. Furthermore, at this $\mathrm{pH}$, very little intensity was observed at $3200 \mathrm{~cm}^{-1}$ and $3400 \mathrm{~cm}^{-1}$, where the interfacial water signal is most dominant (Fig. 1a). Instead, broad features were detected at lower and higher wavenumbers, mainly at $\sim 3000 \mathrm{~cm}^{-}$ ${ }^{1}$ and $\sim 3500 \mathrm{~cm}^{-1}$. 

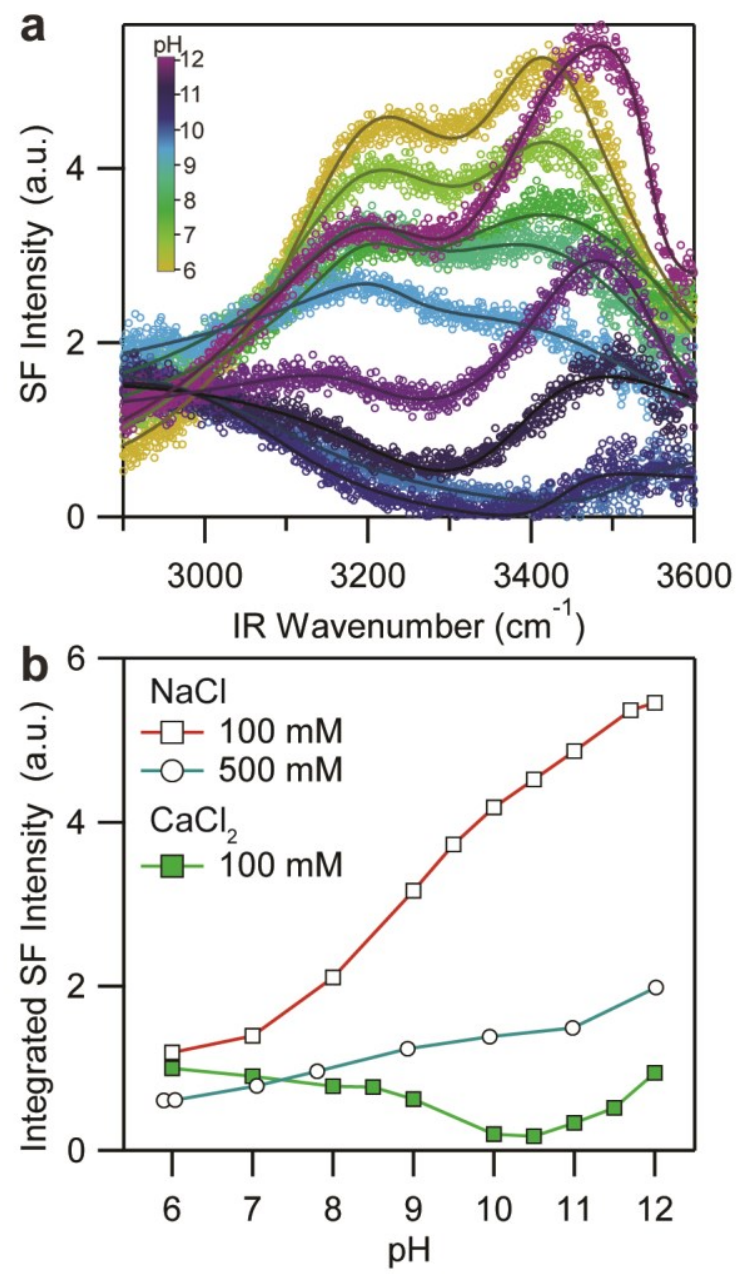

Fig. 1 a) $p p p$-VSF spectra at the silica/aqueous calcium chloride $(100 \mathrm{mM}$, with $10 \mathrm{mM} \mathrm{NaCl})$ interface over a $\mathrm{pH}$ range extending from 6 to 12 collected with the Edmonton SF spectrometer. Lines are guides to the eye. b) Integrated SF intensity between 2900 to $3600 \mathrm{~cm}^{-1}$ from silica in contact with solutions containing $\mathrm{CaCl}_{2}(100 \mathrm{mM}$, with $10 \mathrm{mM} \mathrm{NaCl})$ and $\mathrm{NaCl}(100 \mathrm{mM}$ and 500 $\mathrm{mM}$ ) in an equivalent $\mathrm{pH}$ range, normalized to a common reference, and thus directly comparable.

As mentioned above, most of the signal at $\sim 3200 \mathrm{~cm}^{-1}$ and $\sim 3400 \mathrm{~cm}^{-1}$ is associated with water molecules in the diffuse double layer, which exhibit some net alignment because of interactions between the surface electric field and their electric dipoles. Therefore, the decrease in intensity can be interpreted by a decline of the absolute surface potential and/or Stern potential, 
with the minimum in intensity corresponding to the charge neutralization, or isoelectric, point (IEP). The subsequent increase in the SF signal at higher $\mathrm{pH}$ (i.e. $\mathrm{pH}>10.5$ ), could then be ascribed to a charge reversal where the surface and Stern layer become positively charged, as has been previously observed, for example, upon adsorption of a cationic surfactant on silica. ${ }^{49}$ This interpretation can be substantiated by streaming potential measurements, which can be used to assess the zeta potential of flat insulating oxide surfaces, such as silica. The zeta potential calculated from the change in streaming potential with varying applied pressure is an estimate of the potential at the Stern layer, which includes the layers of dehydrated and hydrated counter ions nearest to the charged surface. ${ }^{50}$

The zeta potential values of a flat silica surface as a function of $\mathrm{pH}$ in the presence of 100 $\mathrm{mM} \mathrm{CaCl}_{2}$ (with $10 \mathrm{mM} \mathrm{NaCl}$ ) and $100 \mathrm{mM} \mathrm{NaCl}$ are shown in Fig. 2. The results provide valuable insight into $\mathrm{pH}$ dependence of the Stern potential. Similar to the VSF results, the pH-dependent zeta potential of the silica/aqueous electrolyte interface in the presence of $\mathrm{CaCl}_{2}$ shows large discrepancies when compared to $\mathrm{NaCl}$ (Fig. 2). 


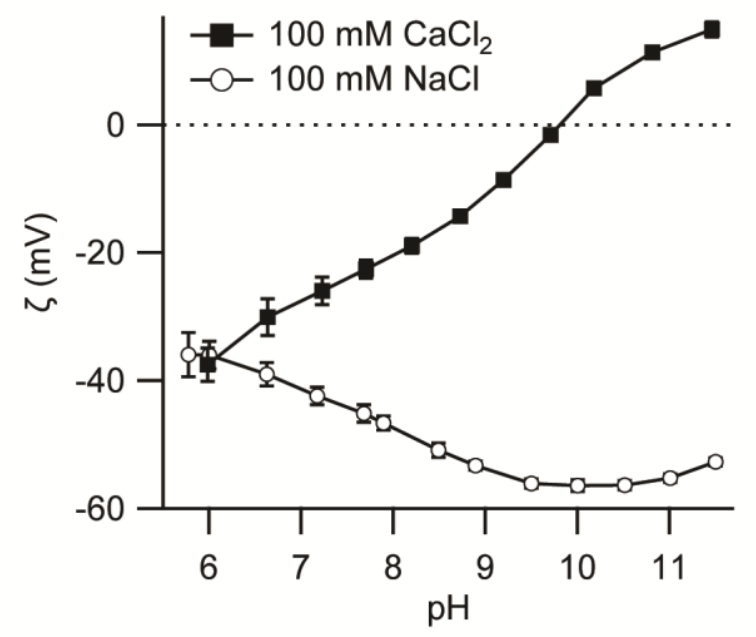

Fig. 2 Zeta potential as a function of $\mathrm{pH}$ calculated from streaming current measurements at the silica/aqueous electrolyte interface in the presence of $100 \mathrm{mM} \mathrm{CaCl}_{2}$ (with $10 \mathrm{mM} \mathrm{NaCl}$ background electrolyte) and $100 \mathrm{mM} \mathrm{NaCl}$. Both silica and salts were from the same suppliers as for the SFG measurements in Fig. 1. Representative traces are shown; duplicates can be found in the ESI.

In the presence of calcium ions, the zeta potential decreased in magnitude with increasing $\mathrm{pH}$, reaching an isoelectric point around $\mathrm{pH} 10$, which is an indication of complete neutralization of surface siloxide sites by the calcium ions present within the Stern layer. The $\mathrm{pH}$ range of the IEP is in accordance with the minimum observed in the SF intensity with silica from the same supplier and the same salt solutions, supporting our interpretation of charge neutralization (i.e. the $\mathrm{pH}$ where the Stern potential reaches zero). At higher $\mathrm{pH}$, the sign of the potential flipped, consistent with the surface becoming net positively charged as inferred from the VSF spectra. In contrast, in the presence of sodium ions the zeta potential increased in magnitude, becoming more negative with increasing $\mathrm{pH}$ (see Fig. 2). The charge overcompensation observed at high $\mathrm{pH}$ arises from an enhanced concentration of calcium species in the interfacial layer that outnumber the negative siloxide sites, eventually leading to a change in the sign of the Stern potential. ${ }^{51}$ This 
charge reversal occurs despite the fact that $\mathrm{SiO}^{-}$groups also increase in numbers at higher $\mathrm{pH}$ upon deprotonation of surface silanol groups.

Near the IEP where we observe a minimum in the SFG intensity it is worth noting the absence of signal intensity from the water modes at $\sim 3200 \mathrm{~cm}^{-1}$ and $3400 \mathrm{~cm}^{-1}$, which are replaced by contributions from interfacial hydroxyl species (or water molecules) with resonant modes at much lower or higher wavenumbers. The lack of intensity in this frequency range lies in contrast to other interfacial systems that exhibited intensity even at the point of zero charge (PZC) or IEP, and may be due to the displacement (or reorientation) of silica hydration waters by calcium ions. ${ }^{52-}$ 55

Additional information on the molecular structure at the silica/aqueous electrolyte interface can be extracted from the SF spectra collected over a broader frequency range, as presented in Fig. 3a. These spectra were measured using the ssp-polarization combination in a total internal reflection (TIR) geometry, which optimizes the sensitivity to modes observed at high wavenumbers. ${ }^{56}$ These experiments were also performed on silica from a different supplier using the Stockholm SF spectrometer. The $p p p$-SFG spectra from the Stockholm SF spectrometer (Fig. S1) and sample generally matched well with the spectra shown in Fig. 2 from the Edmonton SF spectrometer within the same spectral window suggesting both silica samples resulted in similar interfacial structures in the presence of calcium. For the $s s p$ spectra for the lowest $\mathrm{pH}$ measured, in addition to the two bands attributed to aligned water molecules at $\sim 3200 \mathrm{~cm}^{-1}$ and $3400 \mathrm{~cm}^{-1}$, a third peak centred at $\sim 3650 \mathrm{~cm}^{-1}$, previously assigned to the $\mathrm{OH}$ stretch of isolated silanols on the underlying silica surface, can also be resolved in the spectra (Fig. 3a). ${ }^{29}$ With increasing pH, the water bands show a similar trend to that observed in the ppp-polarization combination (Fig. 1a and Fig. S1 in ESI), displaying a minimum in SF intensity at slightly higher $\mathrm{pH}$ between $\mathrm{pH} 10.5$ and 
11. This substantiates the interpretation that the decrease in intensity results indeed from a reduction in the number of aligned water molecules in the diffuse double layer, rather than a change in their orientation. However, concomitant with the decrease in the signal of the $\sim 3400 \mathrm{~cm}^{-1}$ band, an enhanced intensity is observed at high frequencies, with the spectral line shapes consistent with two closely overlapping modes having opposite phases, as it is apparent from the congruous fitting of spectra collected in both the $s s p$ and ppp polarization combinations (see below and ESI). The first is the isolated $\mathrm{SiOH}$ stretch centred at $\sim 3650 \mathrm{~cm}^{-1},{ }^{29}$ which at low $\mathrm{pH}$ is observed as a peak, but as a dip in the spectrum at more basic conditions. The second overlapping band centred at $\sim 3610 \mathrm{~cm}^{-1}$ is assigned to the $\mathrm{OH}$ stretch of adsorbed $\mathrm{Ca}(\mathrm{OH})^{+}$species. The peak position of $\mathrm{Ca}(\mathrm{OH})^{+}$cation is close in range to the fundamental $\mathrm{OH}$ stretching mode of $\mathrm{Ca}(\mathrm{OH})_{2}$ reported at 3645 and $3620 \mathrm{~cm}^{-1}$ in IR and Raman spectra, respectively. ${ }^{57-58}$ Moreover, the band is slightly redshifted and broader than the $\mathrm{Ca}-\mathrm{OH}$ stretch at the $\mathrm{CaF}_{2}$ surface previously observed at $\sim 3657 \mathrm{~cm}^{-}$ ${ }^{1}$ using VSFS. ${ }^{59}$ The increased linewidth is indicative of a greater structural heterogeneity when compared to the crystalline calcium fluoride interface. ${ }^{59}$ 

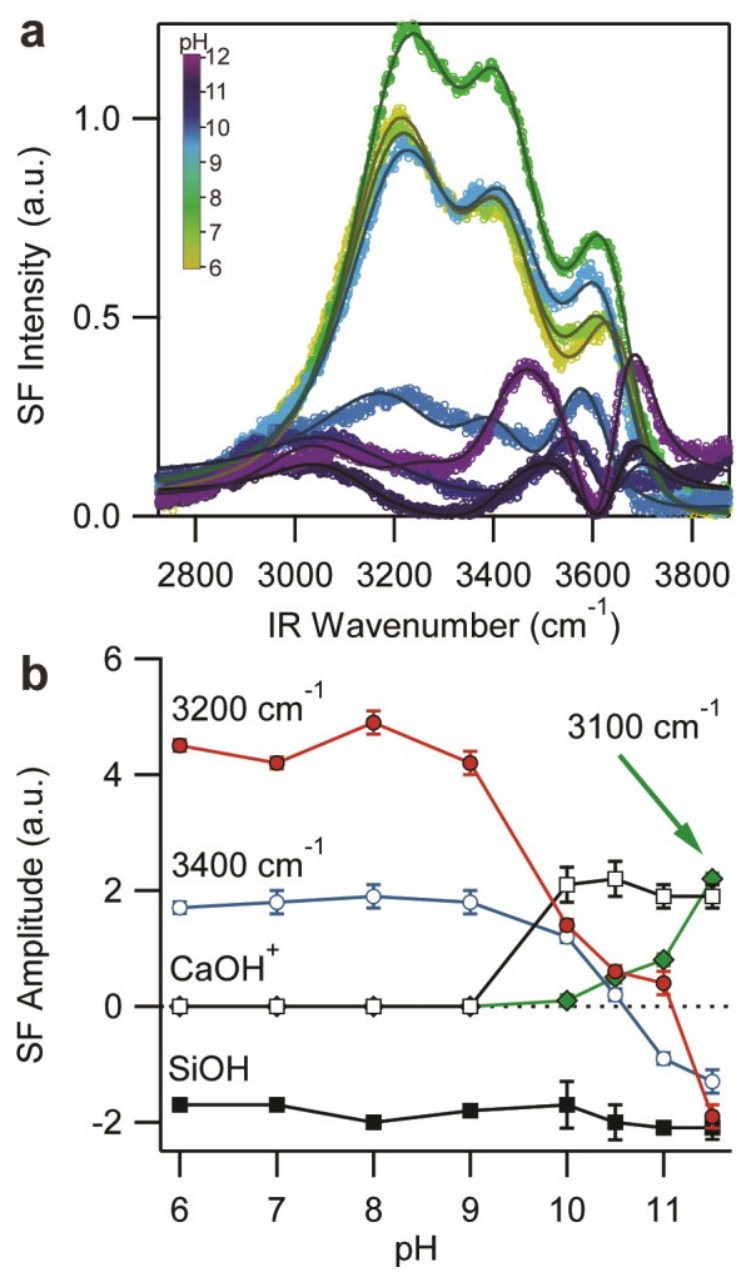

Fig. 3 a) $s s p$-VSF spectra from $2725-3875 \mathrm{~cm}^{-1}$ at the silica/aqueous calcium chloride (100 $\left.\mathrm{mM}\right)$ interface from $\mathrm{pH} 6$ to 11.5 collected with the Stockholm SF spectrometer at an extended frequency range in a gas-tight measuring cell at $22{ }^{\circ} \mathrm{C}$. The similar trends observed when compared to the results in Fig. 1a, provide evidence of the reproducibility of the measured effects, using fused silica substrates from alternative suppliers as well as the potential effects of $\mathrm{CO}_{2}$ dissolved in solution (see Experimental methods for details). The smoothed lines are fits to the spectra using a convolution of Lorentzian and Gaussian line shapes. b) Fitted amplitudes for selected bands as a function of $\mathrm{pH}$. Note that a negative amplitude indicates an $\mathrm{OH}$ directed on average away from the silica surface (see text and ESI for details). 
Further insight into the contributions of the different overlapping and interfering bands can be obtained by fitting the spectra to a convolution of Lorentzian and Gaussian line shapes (see ESI for details). The fitted amplitudes as a function of $\mathrm{pH}$ of the main contributing bands are presented in Fig. 3b. Several interesting aspects can be discerned. The sign gives an indication of the absolute polar orientation of the contributing $\mathrm{OH}$ groups, being positive or negative when facing towards or away from the silica surface, respectively. ${ }^{26,60}$ The amplitude of the $\sim 3400 \mathrm{~cm}^{-1}$ band linked to water molecules in the diffuse layer changes from positive at low $\mathrm{pH}$ to negative at high $\mathrm{pH}$, passing through zero roughly at $\mathrm{pH} 10.5$. The change in sign is consistent with a flip of the effective Stern potential (i.e., from negative to positive). On the other hand, the amplitude of the peak at $\sim 3650 \mathrm{~cm}^{-1}$ linked to the isolated silanols is negative ( $\mathrm{Si}-\mathrm{OH}$ directed away from the interface), and largely independent of the solution $\mathrm{pH}$. Previous work has indicated that isolated silanols have a high $\mathrm{pKa}$ and remain protonated even at $\mathrm{pH}>11,{ }^{29}$ at least at room temperature. ${ }^{34} \mathrm{We}$ note that the isolated silanol band has been assigned by others to water molecules interacting with hydrophobic patches at the silica interface. ${ }^{61}$ However, such an interpretation is inconsistent with the results presented here. In contrast, the amplitude of the band assigned to $\mathrm{Ca}(\mathrm{OH})^{+}$at $\sim 3610 \mathrm{~cm}^{-}$ 1, which becomes apparent from $\mathrm{pH} 10$, has opposite sign, indicating that the cation's $\mathrm{OH}$ is directed towards the solid surface.

At $\mathrm{pH} \geq 10$ we note the appearance of an additional broad band centred at $\sim 3100 \mathrm{~cm}^{-1}$. Given its relatively low frequency, we suggest it arises from the $\mathrm{OH}$ stretch of the first solvation shell water molecules strongly hydrogen bonded to the $\mathrm{OH}^{-}$ion in basic solutions, as previously proposed. ${ }^{62-65}$ It is also consistent with a recent SF study at the silica/aqueous interface with varying $\mathrm{KOH}_{(\mathrm{aq})}$ concentrations, where a distinct shoulder at $3000 \mathrm{~cm}^{-1}$ was attributed to the strong 
hydrogen bonding interaction between the surface water molecules and $\mathrm{OH}^{-} ;{ }^{-66}$ however the contributions from intra- and intermolecular coupling of this mode were not determined. ${ }^{26,67-70}$

As mentioned in the introductory statements, the mechanism of charge reversal on silica and other mineral surfaces by divalent ions has been extensively discussed. ${ }^{1-15}$ Although, charge reversal had been invoked as an explanation for the behavior of silica colloids under certain conditions, ${ }^{1-11}$ the main experimental evidence to test theories of overcharging relied on streaming current measurements on silica at $\mathrm{pH} 7.5$ with increasing calcium or magnesium concentrations. ${ }^{2}$ The authors found that the streaming current reached zero (i.e. the IEP) upon addition of $400 \mathrm{mM}$ calcium chloride and $340 \mathrm{mM}$ magnesium chloride. These results have been qualitatively and quantitatively reproduced using Monte Carlo simulation models that incorporate ion-ion correlations, indicating that such interactions alone could be used to explain overcharging. ${ }^{5}$ However, charge reversal has also been explained for a related aluminosilicate, mica, by only considering dispersion interactions between the ions in solution and surface sites and neglecting ion-ion interactions. ${ }^{4}$ Both theoretical models disregard any specific interactions between the ion and the surface. This includes the formation of hydrolyzed chemical complexes that despite having a lower charge valency, can dominate surface interactions. ${ }^{8}$ For calcium, hydrolysis of the aqueous species yields $\mathrm{Ca}(\mathrm{OH})^{+}$, which we have experimentally proven to preferentially adsorb to the surface. In contrast, SF signal from water molecules hydrating $\mathrm{Ca}^{2+}$ in the Stern layer can not readily be detected owing to the more centrosymmetric structure of the hydrated cation.

We now offer an interpretation for the $\mathrm{pH}$-dependent behavior of the silica/aqueous calcium chloride interface based on the discussion above. At pH 6 the SF signal at $3200 \mathrm{~cm}^{-1}$ and $3400 \mathrm{~cm}^{-1}$ primarily arises from water molecules in the diffuse layer partly aligned by the Stern potential (purple gradient and arrow depicted in Fig. 4), with some contributions from water 
molecules directly interacting with the silica surface (dark purple area in Fig. 4). Isolated silanols detected at $\sim 3650 \mathrm{~cm}^{-1}$ remain protonated in the whole $\mathrm{pH}$ range considered, making them a useful reference. However, as the solution $\mathrm{pH}$ is increased other types of silanols (i.e. vicinal, geminal and/or hydrogen bonded) further deprotonate causing more calcium ions to concentrate at the interface owing to the more negative surface potential. These ions screen the static electric field, thereby decreasing the magnitude of the Stern potential. At $\mathrm{pH} \sim 10.5$ near where the isoelectric point is reached, minimal contributions from water in the original bonded interfacial layer are observed. This lack of signal near the IEP is much more significant than what has been observed in the presence of monovalent cations such as sodium when the water SF intensity passes through a minimum upon changing the $\mathrm{pH}$ (i.e. $\mathrm{pH}$ between 3 and 7, depending on ionic strength). ${ }^{25,31,37}$ We speculate that the strong hydration energy of calcium ${ }^{71}$ allows the divalent ions to remain centrosymmetrically hydrated (i.e. non VSF active) as they approach the surface and displace the hydration layer on silica. This could account for the lack of intensity at $3200 \mathrm{~cm}^{-1}$. Concurrent with charge neutralization upon increasing $\mathrm{pH}$, hydrolyzed calcium cations adsorb (or form) at the surface, with their $\mathrm{OH}$ groups oriented, on average, opposite to those from the underlying isolated silanol functionalities. These observations are consistent with recent MD studies, which show that $\mathrm{Ca}(\mathrm{OH})^{+}$can form at the silica surface upon deprotonation of one of the water molecules in the hydration shell of the $\mathrm{Ca}^{2+}$ cation. ${ }^{72}$ The presence of this hydrolyzed calcium species suggests that chemical interactions must also be considered in addition to ion correlation or dispersion interactions to accurately model the surface. 

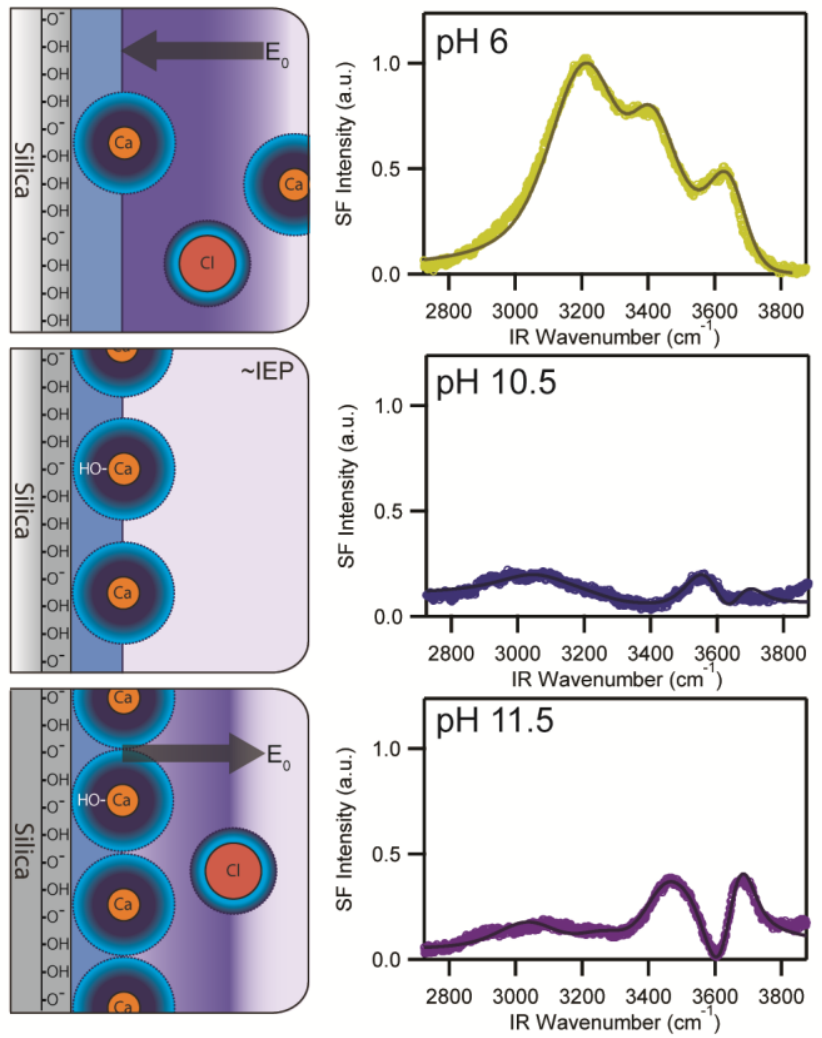

Fig. 4 Molecular representation of the silica/aqueous calcium chloride interface and corresponding ssp-VSF spectra measured at selected $\mathrm{pH}$ values. Smooth lines are the fits to the spectra.

As the $\mathrm{pH}$ is increased beyond the IEP (i.e. from $\mathrm{pH} \sim 10.5$ to $\mathrm{pH} 11.5$ ), the spectral fits reveal a change in sign of the amplitude for both the modes at 3200 and $3400 \mathrm{~cm}^{-1}$. This increase in intensity is consistent with charge inversion due to an excess of calcium ions in the Stern layer leading to a positive Stern potential. Such a charge reversal should align water molecules in the diffuse layer in the opposite orientation to that observed at lower $\mathrm{pH}$ where the Stern potential was negative. Interestingly, while the water bands change in magnitude above $\mathrm{pH} 10$, the amplitude of the $\mathrm{Ca}(\mathrm{OH})^{+}$mode remains approximately constant, suggesting that ion-ion correlations and ionsite dispersion interactions contribute more to the overcharging than specific surface interactions. To verify that indeed the amount of $\mathrm{Ca}(\mathrm{OH})^{+}$remained constant and was not simply reorienting as 
the $\mathrm{pH}$ was increased, we also analyzed the corresponding $p p p$-VSF spectra, which revealed that the $\mathrm{Ca}^{+}-\mathrm{OH}$ mode follows a similar $\mathrm{pH}$-dependent trend (see corresponding spectra and fitting parameters in the ESI).

\section{CONCLUSIONS}

In summary, in the presence of millimolar concentrations of calcium chloride, the electric double layer of the negatively charged silica surface is effectively neutralized upon increasing the $\mathrm{pH}$ to $\sim 10$ and becomes net positive at higher $\mathrm{pH}$. This was demonstrated by both streaming potential and VSF measurements. The charge neutralization and overcompensation are attributed to the adsorption of calcium cations in hydrated and hydroxide forms, with the latter being directly observed in the VSF spectra. However, at high $\mathrm{pH}$, the amount of $\mathrm{Ca}(\mathrm{OH})^{+}$remains constant, indicating that both specific adsorption and ion-ion correlations contribute to silica overcharging. The results also reveal that the interfacial structure of the Stern layer is highly interdependent on the $\mathrm{pH}$, ion valency, and ion concentration.

\section{EXPERIMENTAL METHODS}

Materials. For the Edmonton studies $\mathrm{CaCl}_{2} .6 \mathrm{H}_{2} \mathrm{O}(99.9965 \%$, Puratronic, Alfa Aesar) and $\mathrm{NaCl}$ (99.99\%, trace metals basis, Alfa Aesar) were used for the preparation of salt solutions. $\mathrm{NaOH}$ pellets (99.99\% semiconductor grade, trace metals basis, Sigma-Aldrich) were used to prepare the titrant for $\mathrm{pH}$ adjustments. $\mathrm{KCl}(99.999 \%$, trace metals basis, Acros Organics) was used to calibrate the SurPASS conductivity meter. Methanol (HPLC grade, Fisher Chemical), sulfuric acid (95.0 - 98.0\%, Caledon Laboratories) and hydrogen peroxide (30\% w/w in $\mathrm{H}_{2} \mathrm{O}$, Sigma-Aldrich) were mixed in a 3:1 ratio and used for piranha cleaning of silica hemispheres and plates. All materials were used without further purification. Ultrapure water $(18.2 \mathrm{M} \Omega \cdot \mathrm{cm})$ was used from a 
Milli-Q Direct 8 Water Purification System (Millipore). For the Stockholm measurements, $\mathrm{CaCl}_{2} .2 \mathrm{H}_{2} \mathrm{O}$ (BioUltra $\left.\geq 99.5 \%\right), \mathrm{NaCl}(99.999 \%$ trace metal basis), and $\mathrm{NaOH}(99.99 \%$ trace metal basis) were obtained from Sigma-Aldrich. $\mathrm{NaCl}$ was baked at $550{ }^{\circ} \mathrm{C}$ for $2 \mathrm{~h}$ before use. Chromosulphuric acid, employed for cleaning the silica hemispheres, was purchased from Merck. In both labs, ultrapure water $(18.2 \mathrm{M} \Omega \bullet \mathrm{cm})$ was used after deionization from a Milli-Q-Plus purification system (Millipore). All experiments were performed with freshly prepared solutions.

Sample Preparation. At the Edmonton lab, the IR-grade fused quartz hemispheres (Almaz Optics, KI, 1-inch diameter) and IR-grade fused quartz windows (Almaz Optics, KI, 2.5 inch diameter, 8 $\mathrm{mm}$ thickness) were copiously rinsed and sonicated in Milli-Q water, methanol, and Milli-Q water again. The silica substrates were then immersed in piranha solution (3:1 mixture of $\mathrm{H}_{2} \mathrm{SO}_{4}$ and $\mathrm{H}_{2} \mathrm{O}_{2}, 1$ hour) and then rinsed thoroughly with Milli-Q water. The silica substrates were then rinsed and sonicated in Milli-Q water, methanol, and Milli-Q water again and finally dried in an oven at $110^{\circ} \mathrm{C}(30 \mathrm{~min})$.

Caution: Piranha solution is corrosive and explosive. Extreme heat is generated upon addition of hydrogen peroxide to sulfuric acid. Never add sulfuric acid to hydrogen peroxide, as hydrogen peroxide concentrations greater than $50 \%$ can be explosive. Piranha solution reacts violently with organics, and therefore organic solvents should not be stored nearby.

At the Stockholm lab, the custom-made IR-grade fused silica (Infrasil 302) hemispheres (CVI, Melles Griot, $10 \mathrm{~mm}$ diameter, and $\mathrm{R}_{\mathrm{a}}$ roughness $<0.5 \mathrm{~nm}$ ), were first sonicated in ethanol, rinsed in Milli-Q water, immersed in chromo-sulfuric acid for 30 minutes, and then copiously rinsed and sonicated in Milli-Q water. The hemispheres are always kept underwater and assembled wet on the sample cell to minimize the adsorption of contaminants. The freshly prepared salt solutions were promptly drawn into gas-tight syringes and transferred into a gas-tight measuring cell, which 
design is described in detail elsewhere. ${ }^{73}$ The procedure limits contact of solutions with $\mathrm{CO}_{2}$ in the air, which helps discard any potential effects of bicarbonate or carbonate ions in our result.

The Edmonton VSF spectrometer. A detailed description of the setup can be found elsewhere ${ }^{25}$, 31,37,74. Briefly, $800 \mathrm{~nm}$ laser pulses were generated from a Spitfire (Spitfire Pro, Spectra-Physics, $1 \mathrm{kHz}, 94 \mathrm{fs}, 3.3 \mathrm{~W}$ ), which was seeded by a MaiTai (Spectra-Physics, $80 \mathrm{MHz}$ ) and pumped by an Empower (Nd:YLF laser, Spectra-Physics), respectively. The regeneratively amplified laser light was passed through a 35/65 beam splitter, with $1 \mathrm{~W}$ passing through a Fabry-Perot Etalon (TecOptics) to produce narrow, picosecond pulses (FWHM $\sim 7 \mathrm{~cm}^{-1}$ ). The $800 \mathrm{~nm}$ picosecond light was then passed through a polarizer (Thorlabs) and a half-wave plate (Thorlabs), before being focused with a BK7 focusing lens (focal length $=500 \mathrm{~mm}$, Thorlabs) onto the sample interface at an incidence angle of $61^{\circ}$ from the surface normal at $\sim 10-20 \mu \mathrm{J} /$ pulse. The remaining two-thirds of the amplified femtosecond output ( $2.3 \mathrm{~W}$ ) was used to pump a noncollinear TOPAS-C/NDFG (Light Conversion) to produce broadband IR light $\left(\mathrm{FWHM} \sim 90 \mathrm{~cm}^{-1}\right)$. This tunable light was then passed through a polarizer (Thorlabs) and a tunable zero-order half-wave plate (Alphalas) before being focused using a $\mathrm{CaF}_{2}$ lens (focal length $=500 \mathrm{~mm}$, Thorlabs) onto the sample interface at an incidence angle of $67^{\circ}$ and $\sim 18 \mu \mathrm{J} /$ pulse The visible and IR laser beams were spatially and temporally overlapped at the interface, generating sum frequency (SF) signal. SF light generated from the sample collected in a reflection geometry was recollimated with a BK7 lens (Thorlabs, focal length $=400 \mathrm{~mm}$ ), and then passed through a half-wave plate (Thorlabs) and a GlanThompson calcite polarizer (Thorlabs) to select $S$ or $P$ polarized light. The polarized light was focused through a BK7 lens (Thorlabs, focal length $=100 \mathrm{~mm}$ ) and passed through a shortpass filter (Thorlabs) before entering a benchtop Imaging Spectrograph (SP-2500, Princeton Instruments, grating 1200 grooves $/ \mathrm{mm}, 500 \mathrm{~nm}$ blaze wavelength). The spectrograph was fitted 
onto a thermoelectrically cooled $\left(-75^{\circ} \mathrm{C}\right)$, back-illuminated, charge-coupled device (CCD) camera (Acton PIXIS 100B CCD digital camera system, 1340 X 100 pixels, 20 X $20 \mu \mathrm{m}$ pixel size, Princeton Instruments). Measurements were carried out at $22 \pm 1{ }^{\circ} \mathrm{C}$.

The sample spectra were collected in $s s p$ and $p p p$ polarization configurations and normalized to the nonresonant spectrum of a gold-coated $(200 \mathrm{~nm}$ ) fused quartz hemisphere (Almaz Optics, KI, 1-inch diameter) collected under the $p p p$ polarization configuration. The IR wavelength was scanned between $2900 \mathrm{~cm}^{-1}$ and $3400 \mathrm{~cm}^{-1}$ in steps of $100 \mathrm{~cm}^{-1}$. The gold-coated hemisphere was then replaced with a freshly cleaned, dry, fused silica hemisphere, and VSF spectra were collected for air, Milli-Q water, $10 \mathrm{mM} \mathrm{NaCl}$, and then $100 \mathrm{mM} \mathrm{CaCl}_{2}+10 \mathrm{mM} \mathrm{NaCl}$ solutions over the $\mathrm{pH}$ range extending between 6 and 12. Solution $\mathrm{pH}$ was adjusted with $\mathrm{NaOH}_{(\mathrm{aq})}$. We included 10 $\mathrm{mM} \mathrm{NaCl}$ background electrolyte to account for changes in sodium concentration upon $\mathrm{pH}$ adjustments. High concentration $\mathrm{NaOH}$ solutions were used such that microliter volumes of titrant were required in order to prevent significant changes to solution volume (aqueous phase in SFG sample cell was $10 \mathrm{~mL}$ ). Intensities were again normalized to that of the $3200 \mathrm{~cm}^{-1}$ mode measured from the silica/10 $\mathrm{mM} \mathrm{NaCl}_{(\mathrm{aq})}$ interface.

The Stockholm VSF spectrometer. Described in detail elsewhere, ${ }^{73}$ it briefly consists of a Ti:Sapphire 90fs, 1kHz, 6W amplifier (Integra C, Amplitude Technologies, France), that is used to pump a HE-TOPAS (Light Conversion, Lithuania) and generate tunable mid-IR femtosecond pulses. A fraction of the amplifier output is also used to generate tunable bandwidth picosecond visible $(\sim 805 \mathrm{~nm})$ pulses in a home-built beam shaper. Measurements are carried out with a spectral resolution $<2 \mathrm{~cm}^{-1}$ and a constant temperature of $22^{\circ} \mathrm{C}$. The main elements of the detection system are a Shamrock SR-303i-B spectrometer (Andor, Ireland) and an EM-CCD camera (Newton DU971N-UVB, Andor, Ireland). The Stockholm spectrometer features a large degree of 
automation, which allows collecting spectra at various angles of incidence (AOI) and in an extended spectral range. In these studies, the AOI for the IR beam was set to $55.0^{\circ}$ with an average pulse energy of $3 \mu \mathrm{J}$, while the visible pulse was set to $70.0^{\circ}$ and $\sim 7 \mu \mathrm{J}$, respectively. All spectra were normalized by the nonresonant SF response of an equivalent gold-coated hemisphere, using a procedure described in detail previously. ${ }^{73}$ None of the spectra presented in this work has been Fresnel-factor corrected.

Zeta potential measurements. The zeta potential studies were carried out on a SurPASS Electrokinetic Analyzer (Anton Paar). The samples were setup using a clamping cell following the standard procedure. However, in place of the polystyrene reference film, we modified an IR-grade fused quartz window with two holes to accommodate the electrodes. This modification of the setup removes the requirement to measure the reference film. On the other side of the channel was an unmodified fused quartz window. Prior to each experiment, the instrument was cleaned at least twice with ultrapure water for $300 \mathrm{~s}$ on each cycle using the connection tube between electrodes. With the clamping cell attached, the instrument was filled with water and a flow check to 500 mbar was performed to confirm linear flow rate with pressure. For each solution or solution $\mathrm{pH}$, the instrument was filled (200 s) and rinsed (500 mbar, $500 \mathrm{~s})$ before each measurement (400 mbar, $20 \mathrm{~s}$ ) which involved a prior rinse (500 mbar, $180 \mathrm{~s}$ ). Measurements were performed in streaming current configuration. The conductivity meter was calibrated to $0.1 \mathrm{M} \mathrm{KCl}$ solution. The zeta potentials were calculated from the streaming current using the following equation ${ }^{50}$

$\zeta=\frac{\mathrm{dU}}{\mathrm{d} \Delta \mathrm{p}} \times \frac{\eta}{\varepsilon \times \varepsilon_{0}} \times \kappa$, eq. 2

where $\zeta$ is the zeta potential, $U_{\mathrm{sp}}$ is the streaming potential, $\Delta \mathrm{p}$ is the change in pressure, $\eta$ is the viscosity of the solution, $\varepsilon$ is the relative permittivity of water, $\varepsilon_{0}$ is the vacuum permittivity, and 
$\kappa$ is the conductivity of the bulk solution. Error bars are the standard deviation from four separate pressure ramps.

\section{ORCID}

Mokhtar Rashwan: 0000-0002-5320-1207

Benjamin Rehl: 0000-0002-5534-6717

Eric Tyrode: 0000-0003-1221-0227

Julianne M. Gibbs: 0000-0001-5819-2306

\section{CONFLICTS OF INTEREST}

There are no conflicts to declare.

\section{ACKNOWLEDGEMENTS}

J.M.G. gratefully acknowledges the Natural Sciences and Engineering Research Council (NSERC) of Canada for an Accelerator Award, the Alfred P. Sloan Foundation for a Research Fellowship, and Petro-Canada for a Young Innovator Award. B.R. and M.R. gratefully acknowledges support from the Alberta/Technical University of Munich International Graduate School for Hybrid Functional Materials (ATUMS-NSERC CREATE) and the University of Alberta Future Energy Systems (Canada First Research Excellence Fund). B.R. also acknowledges the NSERC of Canada for a Canadian Graduate Scholarship, and the Queen Elizabeth II Graduate Scholarship. A.S. and E.T. acknowledge financial support from the Swedish Foundation for Strategic Research (SSF) through the program "Future Research Leaders-5." M.S.A., Q.L. and H.Z. acknowledge the Canadian Centre for Clean Coal/Carbon and Mineral Processing Technologies at the University of Alberta for funding. 


\section{NOTES AND REFERENCES}

1. Lorenz, C. D.; Travesset, A., Charge Inversion of Divalent lonic Solutions in Silica Channels. Phys. Rev. E 2007, 75 (6), 061202.

2. $\quad$ van der Heyden, F. H. J.; Stein, D.; Besteman, K.; Lemay, S. G.; Dekker, C., Charge Inversion at High Ionic Strength Studied by Streaming Currents. Phys. Rev. Lett. 2006, 96 (22), 224502.

3. Shklovskii, B. I., Screening of a Macroion by Multivalent lons: Correlation-Induced Inversion of Charge. Phys. Rev. E 1999, 60 (5), 5802-5811.

4. Parsons, D. F.; Ninham, B. W., Charge Reversal of Surfaces in Divalent Electrolytes: The Role of Ionic Dispersion Interactions. Langmuir 2010, 26 (9), 6430-6436.

5. Labbez, C.; Jönsson, B.; Skarba, M.; Borkovec, M., Ion-lon Correlation and Charge Reversal at Titrating Solid Interfaces. Langmuir 2009, 25 (13), 7209-7213.

6. Besteman, K.; Zevenbergen, M. A. G.; Heering, H. A.; Lemay, S. G., Direct Observation of Charge Inversion by Multivalent lons as a Universal Electrostatic Phenomenon. Phys. Rev. Lett. 2004, 93 (17), 170802.

7. Piontek, S. M.; Tuladhar, A.; Marshall, T.; Borguet, E., Monovalent and Divalent Cations at the $\alpha-$ $\mathrm{Al}_{2} \mathrm{O}_{3}(0001) /$ Water Interface: How Cation Identity Affects Interfacial Ordering and Vibrational Dynamics. J. Phys. Chem. C 2019, 123 (30), 18315-18324.

8. de Vos, W. M.; Lindhoud, S., Overcharging and Charge Inversion: Finding the Correct Explanation(s). Adv. Colloid Interfac. 2019, 274, 102040.

9. Wiese, G. R.; Healy, T. W., Adsorption of Al(III) at the TiO2 - H2O Interface. J. Colloid Interf. Sci. 1975, 51 (3), 434-442.

10. James, R. O.; Healy, T. W., Adsorption of Hydrolyzable Metal lons at the Oxide-Water Interface. II. Charge Reversal of SiO2 and TiO2 Colloids by Adsorbed Co(II), La(III), and Th(IV) as Model Systems. J. Colloid Interf. Sci. 1972, 40 (1), 53-64.

11. Jiménez, M. L.; Delgado, Á. V.; Lyklema, J., Hydrolysis versus Ion Correlation Models in Electrokinetic Charge Inversion: Establishing Application Ranges. Langmuir 2012, 28 (17), 6786-6793.

12. Lyklema, J., Overcharging, charge reversal: Chemistry or physics? Colloids Surf. A Physicochem. Eng. Asp. 2006, 291 (1), 3-12.

13. Mpofu, P.; Addai-Mensah, J.; Ralston, J., Influence of Hydrolyzable Metal lons on the Interfacial Chemistry, Particle Interactions, and Dewatering Behavior of Kaolinite Dispersions. J. Colloid Interf. Sci. 2003, 261 (2), 349-359.

14. Chemeda, Y. C.; Deneele, D.; Christidis, G. E.; Ouvrard, G., Influence of Hydrated Lime on the Surface Properties and Interaction of Kaolinite Particles. Appl. Clay Sci. 2015, 107, 1-13.

15. Labbez, C.; Nonat, A.; Pochard, I.; Jönsson, B., Experimental and Theoretical Evidence of Overcharging of Calcium Silicate Hydrate. J. Colloid Interf. Sci. 2007, 309 (2), 303-307.

16. Legg, B. A.; Baer, M. D.; Chun, J.; Schenter, G. K.; Huang, S.; Zhang, Y.; Min, Y.; Mundy, C. J.; De Yoreo, J. J., Visualization of Aluminum lons at the Mica Water Interface Links Hydrolysis State to Surface Potential and Particle Adhesion. J. Am. Chem. Soc. 2020.

17. Sartin, M. M.; Sung, W.; Nihonyanagi, S.; Tahara, T., Molecular Mechanism of Charge Inversion Revealed by Polar Orientation of Interfacial Water Molecules: A Heterodyne-Detected Vibrational Sum Frequency Generation Study. J. Chem. Phys. 2018, 149 (2), 024703.

18. Chang, H.; Ohno, P. E.; Liu, Y.; Lozier, E. H.; Dalchand, N.; Geiger, F. M., Direct Measurement of Charge Reversal on Lipid Bilayers Using Heterodyne-Detected Second Harmonic Generation Spectroscopy. J. Phys. Chem. B 2020, 124 (4), 641-649.

19. Dermont, G.; Bergeron, M.; Mercier, G.; Richer-Laflèche, M., Soil Washing for Metal Removal: A Review of Physical/Chemical Technologies and Field Applications. J. Hazard. Mater. 2008, 152 (1), 1-31. 
20. Cruz, N.; Peng, Y.; Wightman, E.; Xu, N., The Interaction of Clay Minerals with Gypsum and its Effects on Copper-Gold Flotation. Miner. Eng. 2015, 77, 121-130.

21. Kasprzyk-Hordern, B., Chemistry of Alumina, Reactions in Aqueous Solution and its Application in Water Treatment. Adv. Colloid Interfac. 2004, 110 (1), 19-48.

22. Chalaturnyk, R. J.; Don Scott, J.; Özüm, B., Management of Oil Sands Tailings. Pet. Sci. Technol. 2002, 20 (9-10), 1025-1046.

23. Farkish, A.; Fall, M., Rapid Dewatering of Oil Sand Mature Fine Tailings Using Super Absorbent Polymer (Sap). Miner. Eng. 2013, 50-51, 38-47.

24. Bourgès-Gastaud, S.; Dolez, P.; Blond, E.; Touze-Foltz, N., Dewatering of Oil Sands Tailings With an Electrokinetic Geocomposite. Miner. Eng. 2017, 100, 177-186.

25. Darlington, A. M.; Jarisz, T. A.; DeWalt-Kerian, E. L.; Roy, S.; Kim, S.; Azam, M. S.; Hore, D. K.; Gibbs, J. M., Separating the $\mathrm{pH}$-Dependent Behavior of Water in the Stern and Diffuse Layers with Varying Salt Concentration. J. Phys. Chem. C 2017, 121, 20229-20241.

26. Myalitsin, A.; Urashima, S.; Nihonyanagi, S.; Yamaguchi, S.; Tahara, T., Water Structure at the Buried Silica/Aqueous Interface Studied by Heterodyne-Detected Vibrational Sum-Frequency Generation. J. Phys. Chem. C 2016, 120 (17), 9357-9363.

27. Isaienko, O.; Nihonyanagi, S.; Sil, D.; Borguet, E., Observation of the Bending Mode of Interfacial Water at Silica Surfaces by Near-Infrared Vibrational Sum-Frequency Generation Spectroscopy of the [Stretch + Bend] Combination Bands. J. Phys. Chem. Lett. 2013, 4 (3), 531-535.

28. Jena, K. C.; Hore, D. K., Variation of Ionic Strength Reveals the Interfacial Water Structure at a Charged Mineral Surface. J. Phys. Chem. C 2009, 113, 15364-15372.

29. Dalstein, L.; Potapova, E.; Tyrode, E., The Elusive Silica/Water Interface: Isolated Silanols Under Water as Revealed by Vibrational Sum Frequency Spectroscopy. Phys. Chem. Chem. Phys. 2017, 19, 1034310349.

30. Ostroverkhov, V.; Waychunas, G. A.; Shen, Y. R., Vibrational Spectra of Water at Water/a-Quartz (0001) Interface. Chem. Phys. Lett. 2004, 386 (1), 144-148.

31. DeWalt-Kerian, E. L.; Kim, S.; Azam, M. S.; Zeng, H.; Liu, Q.; Gibbs, J. M., pH-Dependent Inversion of Hofmeister Trends in the Water Structure of the Electrical Double Layer. J. Phys. Chem. Lett. 2017, 8 (13), 2855-2861.

32. Hore, D. K.; Tyrode, E., Probing Charged Aqueous Interfaces Near Critical Angles: Effect of Varying Coherence Length. J. Phys. Chem. C 2019, 123 (27), 16911-16920.

33. Schaefer, J.; Gonella, G.; Bonn, M.; Backus, E. H. G., Surface-Specific Vibrational Spectroscopy of the Water/Silica Interface: Screening and Interference. Phys. Chem. Chem. Phys. 2017, 19 (25), 1687516880.

34. Azam, M. S.; Cai, C.; Gibbs, J. M.; Tyrode, E.; Hore, D. K., Silica Surface Charge Enhancement at Elevated Temperatures Revealed by Interfacial Water Signals. J. Am. Chem. Soc. 2020, 142 (2), 669-673.

35. Flores, S. C.; Kherb, J.; Konelick, N.; Chen, X.; Cremer, P. S., The Effects of Hofmeister Cations at Negatively Charged Hydrophilic Surfaces. J. Phys. Chem. C 2012, 116 (9), 5730-5734.

36. Lovering, K. A.; Bertram, A. K.; Chou, K. C., New Information on the Ion-Identity-Dependent Structure of Stern Layer Revealed by Sum Frequency Generation Vibrational Spectroscopy. J. Phys. Chem. C 2016, 120 (32), 18099-18104.

37. Rehl, B.; Rashwan, M.; DeWalt-Kerian, E. L.; Jarisz, T. A.; Darlington, A. M.; Hore, D. K.; Gibbs, J. M., New Insights into $\chi(3)$ Measurements: Comparing Nonresonant Second Harmonic Generation and Resonant Sum Frequency Generation at the Silica/Aqueous Electrolyte Interface. J. Phys. Chem. C 2019, 123 (17), 10991-11000.

38. Lambert, A. G.; Davies, P. B.; Neivandt, D. J., Implementing the Theory of Sum Frequency Generation Vibrational Spectroscopy: A Tutorial Review. Appl. Spectrosc. Rev. 2005, 40 (2), 103-145. 
39. Jena, K. C.; Covert, P. A.; Hore, D. K., The Effect of Salt on the Water Structure at a Charged Solid Surface: Differentiating Second- and Third-Order Nonlinear Contributions. J. Phys. Chem. Lett. 2011, 2, 1056-1061.

40. Ohno, P. E.; Wang, H.-f.; Geiger, F. M., Second-Order Spectral Lineshapes from Charged Interfaces. Nat. Commun. 2017, 8 (1), 1032.

41. Ohno, P. E.; Wang, H.-f.; Paesani, F.; Skinner, J. L.; Geiger, F. M., Second-Order Vibrational Lineshapes from the Air/Water Interface. J. Phys. Chem. A 2018, 122 (18), 4457-4464.

42. Wen, Y.-C.; Zha, S.; Liu, X.; Yang, S.; Guo, P.; Shi, G.; Fang, H.; Shen, Y. R.; Tian, C., Unveiling Microscopic Structures of Charged Water Interfaces by Surface-Specific Vibrational Spectroscopy. Phys. Rev. Lett. 2016, 116 (1), 016101.

43. Gonella, G.; Lütgebaucks, C.; de Beer, A. G. F.; Roke, S., Second Harmonic and Sum-Frequency Generation from Aqueous Interfaces Is Modulated by Interference. J. Phys. Chem. C 2016, 120 (17), 91659173.

44. Tyrode, E.; Corkery, R., Charging of Carboxylic Acid Monolayers with Monovalent lons at Low lonic Strengths: Molecular Insight Revealed by Vibrational Sum Frequency Spectroscopy. J. Phys. Chem. C 2018, 122 (50), 28775-28786.

45. Du, Q.; Freysz, E.; Shen, Y. R., Vibrational Spectra of Water Molecules at Quartz/Water Interfaces. Phys. Rev. Lett. 1994, 72, 238-241.

46. Yang, Z.; Li, Q.; Chou, K. C., Structures of Water Molecules at the Interfaces of Aqueous Salt Solutions and Silica:Cation Effects. J. Phys. Chem. C 2009, 113, 8201-8205.

47. Joutsuka, T.; Hirano, T.; Sprik, M.; Morita, A., Effects of Third-Order Susceptibility in Sum Frequency Generation Spectra: A Molecular Dynamics Study in Liquid Water. Phys. Chem. Chem. Phys. 2018, 20 (5), 3040-3053.

48. Ong, S.; Zhao, X.; Eisenthal, K. B., Polarization of Water Molecules at a Charged Interface: Second Harmonic Studies of the Silica/Water Interface. Chem. Phys. Lett. 1992, 191, 327-335.

49. Tyrode, E.; Rutland, M. W.; Bain, C. D., Adsorption of CTAB on Hydrophilic Silica Studied by Linear and Nonlinear Optical Spectroscopy. J. Am. Chem. Soc. 2008, 130 (51), 17434-45.

50. Luxbacher, T.; Anton Paar Gmb, H., The Zeta Potential for Solid Surface Analysis : A Practical Guide to Streaming Potential Measurement. Anton Paar GmbH: Austria, 2014.

51. Brown, M. A.; Goel, A.; Abbas, Z., Effect of Electrolyte Concentration on the Stern Layer Thickness at a Charged Interface. Angew. Chem. Int. Ed. 2016, 55, 3790 - 3794.

52. Sung, J.; Zhang, L.; Tian, C.; Shen, Y. R.; Waychunas, G. A., Effect of pH on the Water $/ \alpha-\mathrm{Al}_{2} \mathrm{O}_{3}(1 \mathrm{IO} 2)$ Interface Structure Studied by Sum-Frequency Vibrational Spectroscopy. J. Phys. Chem. C 2011, 115 (28), 13887-13893.

53. Yeganeh, M. S.; Dougal, S. M.; Pink, H. S., Vibrational Spectroscopy of Water at Liquid/Solid Interfaces: Crossing the Isoelectric Point of a Solid Surface. Phys. Rev. Lett. 1999, 83 (6), 1179-1182.

54. Kataoka, S.; Gurau, M. C.; Albertorio, F.; Holden, M. A.; Lim, S.-M.; Yang, R. D.; Cremer, P. S., Investigation of Water Structure at the $\mathrm{TiO}_{2}$ /Aqueous Interface. Langmuir 2004, 20 (5), 1662-1666.

55. Dreier, L. B.; Bernhard, C.; Gonella, G.; Backus, E. H. G.; Bonn, M., Surface Potential of a Planar Charged Lipid-Water Interface. What Do Vibrating Plate Methods, Second Harmonic and Sum Frequency Measure? J. Phys. Chem. Lett. 2018, 9 (19), 5685-5691.

56. Liljeblad, J. F. D.; Tyrode, E., Vibrational Sum Frequency Spectroscopy Studies at Solid/Liquid Interfaces: Influence of the Experimental Geometry in the Spectral Shape and Enhancement. J. Phys. Chem. C 2012, 116 (43), 22893-22903.

57. Weckler, B.; Lutz, H. D., Near-Infrared Spectra of $\mathrm{M}(\mathrm{OH}) \mathrm{Cl}(\mathrm{M}=\mathrm{Ca}, \mathrm{Cd}, \mathrm{Sr}), \mathrm{Zn}(\mathrm{OH}) \mathrm{F}, \mathrm{\gamma}-\mathrm{Cd}(\mathrm{OH}) 2$, $\mathrm{Sr}(\mathrm{OH}) 2$, and Brucite-Type Hydroxides $\mathrm{M}(\mathrm{OH}) 2(\mathrm{M}=\mathrm{Mg}, \mathrm{Ca}, \mathrm{Mn}, \mathrm{Fe}, \mathrm{Co}, \mathrm{Ni}, \mathrm{Cd})$. Spectrochim. Acta. A 1996, 52 (11), 1507-1513. 
58. Lutz, H. D.; Möller, H.; Schmidt, M., Lattice Vibration Spectra. Part LXXXII. Brucite-type Hydroxides $\mathrm{M}(\mathrm{OH}) 2(\mathrm{M}=\mathrm{Ca}, \mathrm{Mn}, \mathrm{Co}, \mathrm{Fe}, \mathrm{Cd})-\mathrm{IR}$ and Raman Spectra, Neutron Diffraction of Fe(OH)2. J. Mol. Struct. 1994, 328, 121-132.

59. Becraft, K. A.; Richmond, G. L., In Situ Vibrational Spectroscopic Studies of the $\mathrm{CaF}_{2} / \mathrm{H}_{2} \mathrm{O}$ Interface. Langmuir 2001, 17 (25), 7721-7724.

60. Urashima, S.-h.; Myalitsin, A.; Nihonyanagi, S.; Tahara, T., The Topmost Water Structure at a Charged Silica/Aqueous Interface Revealed by Heterodyne-Detected Vibrational Sum Frequency Generation Spectroscopy. J. Phys. Chem. Lett. 2018, 9 (14), 4109-4114.

61. Cyran, J. D.; Donovan, M. A.; Vollmer, D.; Siro Brigiano, F.; Pezzotti, S.; Galimberti, D. R.; Gaigeot, M.-P.; Bonn, M.; Backus, E. H. G., Molecular Hydrophobicity at a Macroscopically Hydrophilic Surface. Proc. Natl. Acad. Sci. 2019, 116 (5), 1520.

62. Mandal, A.; Tokmakoff, A., Vibrational Dynamics of Aqueous Hydroxide Solutions Probed using Broadband 2DIR Spectroscopy. J. Chem. Phys. 2015, 143 (19), 194501.

63. Mandal, A.; Ramasesha, K.; De Marco, L.; Tokmakoff, A., Collective Vibrations of Water-solvated Hydroxide lons Investigated with Broadband 2DIR Spectroscopy. J. Chem. Phys. 2014, 140 (20), 204508.

64. Chen, B.; Ivanov, I.; Park, J. M.; Parrinello, M.; Klein, M. L., Solvation Structure and Mobility Mechanism of OH-: A Car-Parrinello Molecular Dynamics Investigation of Alkaline Solutions. J. Phys. Chem. B 2002, 106 (46), 12006-12016.

65. Imamura, T.; Ishiyama, T.; Morita, A., Molecular Dynamics Analysis of $\mathrm{NaOH}$ Aqueous Solution Surface and the Sum Frequency Generation Spectra: Is Surface OH- Detected by SFG Spectroscopy? J. Phys. Chem. C 2014, 118 (50), 29017-29027.

66. Lyu, Y.; Wang, Y.; Wang, S.; Liu, B.; Du, H., Potassium Hydroxide Concentration-Dependent Water Structure on the Quartz Surface Studied by Combining Sum-Frequency Generation (SFG) Spectroscopy and Molecular Simulations. Langmuir 2019, 35 (36), 11651-11661.

67. Buch, V., Molecular Structure and OH-Stretch Spectra of Liquid Water Surface. J. Phys. Chem. B 2005, 109 (38), 17771-17774.

68. Torii, H., Time-Domain Calculations of the Polarized Raman Spectra, the Transient Infrared Absorption Anisotropy, and the Extent of Delocalization of the OH Stretching Mode of Liquid Water. J. Phys. Chem. A 2006, 110 (30), 9469-9477.

69. Auer, B. M.; Skinner, J. L., IR and Raman spectra of liquid water: Theory and interpretation. J. Chem. Phys. 2008, 128 (22), 224511.

70. Sovago, M.; Kramer Campen, R.; Bakker, H. J.; Bonn, M., Hydrogen Bonding Strength of Interfacial Water Determined with Surface Sum-Frequency Generation. Chem. Phys. Lett. 2009, 470 (1), 7-12.

71. Tansel, B.; Sager, J.; Rector, T.; Garland, J.; Strayer, R. F.; Levine, L.; Roberts, M.; Hummerick, M.; Bauer, J., Significance of Hydrated Radius and Hydration Shells on lonic Permeability during Nanofiltration in Dead End and Cross Flow Modes. Sep. Purif. Technol. 2006, 51 (1), 40-47.

72. Wang, X.; Liu, W.; Duan, H.; Wang, B.; Han, C.; Wei, D., The Adsorption Mechanism of Calcium lon on Quartz (101) Surface: A DFT Study. Powder Technol. 2018, 329, 158-166.

73. Liljeblad, J. F. D.; Tyrode, E., Vibrational Sum Frequency Spectroscopy Studies at Solid/Liquid Interfaces: Influence of the Experimental Geometry in the Spectral Shape and Enhancement. J. Phys. Chem. C 2012, 116 (43), 22893-22903.

74. Rehl, B.; Li, Z.; Gibbs, J. M., Influence of High pH on the Organization of Acetonitrile at the Silica/Water Interface Studied by Sum Frequency Generation Spectroscopy. Langmuir 2018, 34 (15), 44454454. 